\title{
Inflammatory lymphangiogenesis in postpartum breast tissue remodeling
}

\author{
Melody A. Swartz \\ Institute for Molecular Engineering, University of Chicago, Chicago, Illinois, USA. Institute of Bioengineering, Ecole Polytechnique Fédérale de Lausanne, Lausanne, Switzerland.
}

\begin{abstract}
Like many cancers, mammary carcinomas use lymphatic vessels to disseminate, and numerous clinical and experimental studies have documented a strong correlation between peritumoral lymphangiogenesis and tumor dissemination. At the same time, many other factors can affect the incidence, invasiveness, and mortality of breast cancer, including lactation history. Although lactation reduces overall cancer risk, patients diagnosed within 5 years of pregnancy have an increased incidence of metastatic disease. In this issue of the $J C l$, Lyons and colleagues demonstrate that postpartum breast tissue remodeling during involution coincides with inflammatory lymphangiogenesis. In mouse models, cyclooxygenase-2 (COX-2) inhibition during involution reduced the risk of cancer metastasis and correlated with decreased lymphangiogenesis. In addition to lymphangiogenesis, COX-2 inhibition reduces many of the immune-suppressive features of the tumor microenvironment, including development of myeloid-derived suppressor cells and regulatory T cells; therefore, these results support the notion that inhibiting COX-2 during lactation weaning may lessen the incidence of breast cancer metastasis.
\end{abstract}

Overall, cyclooxygenase-2-driven (COX-2driven) $\mathrm{PGE}_{2}$ upregulation is thought to help control cytotoxic immune responses in later stages of inflammation; as such, it is also expressed in chronic infection and autoimmunity (5). The major actions of COX-2-dependent $\mathrm{PGE}_{2}$ expression on leukocytes and stromal cells tend to be suppressive, and in the tumor microenvironment, this pathway drives development of myeloid-derived suppressor cells, regulatory $\mathrm{T}$ cells, and alternatively activated macrophages. In dendritic cells, $\mathrm{PGE}_{2}$ upregulates suppressive factors, like IL-10 and indoleamine 2,3-dioxygenase (IDO), leading to dysfunctional $\mathrm{T}$ cell activation. Therefore, since these inflammatory events in the tumor microenvironment are coincident with lymphangiogenesis, teasing out the mechanisms by which lymphangiogenesis specifically contributes to cancer metastasis is inherently difficult.

\section{Lymphangiogenesis in cancer, inflammation, and tissue remodeling}

Lymphatic vessels are common routes for tumor cell metastasis, and sentinel lymph node metastasis is a major prognostic indicator of breast cancer outcome. Since the identification of lymphatic-specific growth factors VEGF-C and VEGF-D and their receptor VEGFR-3, numerous studies have demonstrated striking correlations between tumor-associated lymphangiogenesis, or peritumoral lymphatic expansion, and metastasis (1). Originally the correlation between lymphangiogenesis and tumor metastasis was attributed to increased accessibility for tumor cell dissemination, but recent studies have shown that tumor-associated lymphangiogenesis provides many immune-suppressive features to the tumor microenvironment and can pacify tumor-specific cytotoxic $\mathrm{T}$ lymphocytes as well as drive deletional tolerance of naive T cells $(2,3)$.

On the other hand, lymphangiogenesis is not specific to the tumor microenvironment and generally accompanies all types of inflammation, particularly in late or chronic stages, including chronic infections, wound healing and tissue remodeling, autoimmune diseases like Crohn's disease, and the resolution phase of acute inflammation $(3,4)$. Lymphangiogenesis is driven by a host of inflammatory cells that secrete VEGF-C, including mast cells, neutrophils, macrophages, activated stromal cells, angiogenic blood endothelium, and $\mathrm{B}$ cells (3). These cells can upregulate their production of VEGF-C or VEGF-D upon exposure to prostaglandin $\mathrm{E} 2\left(\mathrm{PGE}_{2}\right)$, which plays many important and complex roles in the tumor microenvironment.

Related Article: p. 3901

Conflict of interest: The author has declared that no conflict of interest exists.

Reference information: J Clin Invest. 2014;124(9):3704-3707. doi:10.1172/JCI77765.

\section{Function and consequences of inflammatory lymphangiogenesis}

During inflammation, lymphangiogenesis often involves hyperplasia of preexisting lymphatic vessels, increased vessel diameters, and decreased organization and patterning. The expanded lymphatic network in inflamed tissues often resembles the vascular plexus of the liver sinusoids or bone marrow vasculature. It remains controversial whether these altered vessels have increased transport functions draining fluid or carrying cells to the lymph node - but several recent studies have suggested other functions of lymphatic expansion. First, as mentioned above, VEGF-C and VEGF-D are triggered in later stages of inflammation. $\mathrm{PGE}_{2}$ is a key driver of inflammatory lymphangiogenesis through its actions on inflammatory cells that secrete VEGF-C and VEGF-D. Moreover, upregulation of $\mathrm{PGE}_{2}$ is considered to be key for the resolution of inflammation and dampening cytotoxic immune responses; therefore, the activated lymphatic endo- 


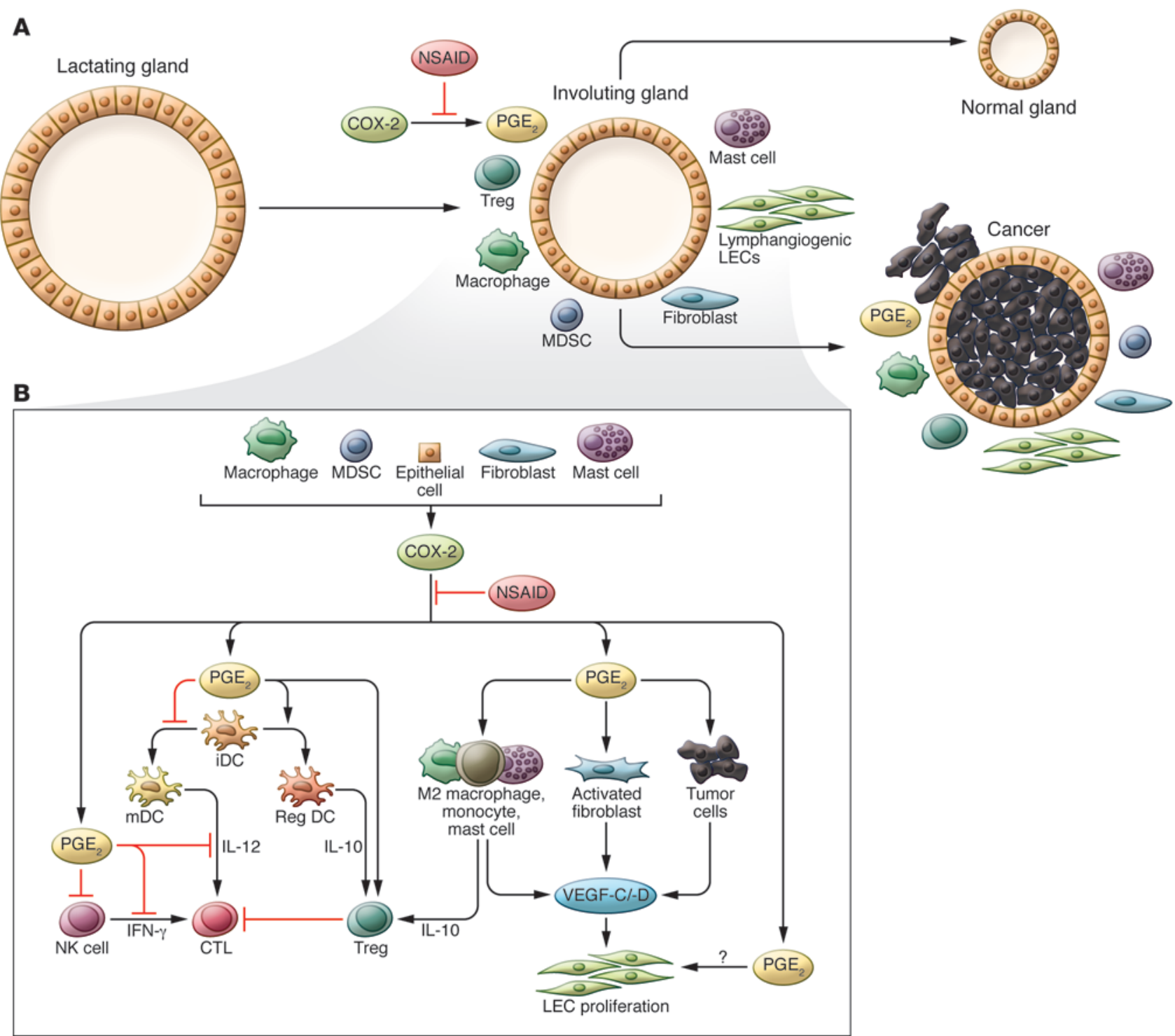

Figure 1. $\mathrm{PGE}_{2}$ is involved in later stages of inflammation and promotes immune-suppressive cell types and lymphangiogenesis through complex and interacting pathways. (A) Common features of the inflammatory microenvironment found in tissue remodeling, wound healing, chronic inflammation, and cancer. Alternatively activated or M2-like macrophages, fibroblasts, epithelial cells, myeloid-derived suppressor cells (MDSCs), and mast cells are among the sources of COX-2 that drive PGE ${ }_{2}$ production. $\mathrm{PCE}_{2}$ drives the upregulation of lymphangiogenic growth factors VEGF-C and VEGF-D by several cell types, including monocytes, mast cells, macrophages, fibroblasts, and tumor cells. PGE ${ }_{2}$ also suppresses both innate and adaptive immune responses through multiple actions, including differentiation of regulatory T cells, suppression of NK cells, and skewing of dendritic cell (DC) phenotypes toward regulatory dendritic cells, which together with monocytes, myeloid-derived suppressor cells, and M2-like macrophages secrete immune-suppressive factors like IL-10, TCF- $\beta$, and IDO. Together, these immune-suppressive features of the PGE inflammation, wound repair, and tissue remodeling (including during mammary gland involution) and for restoring tissue homeostasis and function while avoiding unwanted autoimmune responses; on the other hand, the same features promote immune escape of malignant cells and thus promote cancer and metastasis. (B) The breast tissue undergoes drastic remodeling during involution and is populated by immune-suppressive cell types and cytokines that help drive lymphangiogenesis. Inhibiting COX-2 during involution may help suppress or prevent this inflammatory microenvironment and therefore risk of metastatic breast cancer in some women, but it remains unknown how inhibiting COX-2 will affect normal involution and restoration of the mammary gland to a homeostatic state. $\mathrm{mDC}$, mature dendritic cell; iDC, immature dendritic cell.

thelium likely serves immune-suppressive roles in such an environment. Second, lymphatic endothelial cells (LECs) have been shown to suppress dendritic cell maturation, secrete suppressive cytokines and factors like IDO, and directly activate naive $\mathrm{T}$ cells for deletional tolerance (3); lymphatic hyperplasia could therefore increase the relative importance of these functions by increasing LEC surface area. Finally, VEGF-C triggers LECs to upregulate CCL21 (6), a lymphoid homing chemokine that attracts not only dendritic cells and naive $\mathrm{T}$ cells but also regulatory $\mathrm{T}$ cells. Thus, while inflammatory cells drive lymphangiogenesis, lymphangiogenesis also alters the local immune cell repertoire.

It was first suggested a decade ago that COX-2 could drive inflammatory or tumor-associated lymphangiogenesis. At this time, activation of the E-prost- 
anoid receptor 1 (EP1) was reported to upregulate VEGF-C in tumor cell lines that overexpressed COX-2 (7), and COX2 expression was positively correlated with VEGF-C expression and lymphatic vessel density in human specimens of lung and breast cancer $(7,8)$. Different EP receptors have been implicated in COX2-driven lymphangiogenesis, depending on the cell type and model used. The diversity of identified EP receptors is not surprising given the host of cell types in the tumor microenvironment (including tumor cells themselves) that can express prostaglandin receptors as well as secrete VEGF-C and VEGF-D. For example, in human lung and breast cancer cell lines, COX-2 overexpression has been shown to stimulate endogenous $\mathrm{PGE}_{2}$-mediated EP1 and EP4 signaling to drive upregulation of VEGF-C and VEGF-D directly by the tumor cells $(7,9)$. In this issue, Lyons et al. report that expression of $\mathrm{PGE}_{2}$ by postpartum tumor cells stimulates LECs directly in an EP2-dependent manner (10), although other studies of tumor-associated stroma implicate EP3 $(11,12)$. In human prostate cancer, EP3 protein levels were positively correlated with lymphatic vessel density (11), and COX2-overexpressing Lewis lung carcinomas drove stromal cell secretion of VEGF-C via EP3 activation (12). Therefore, $\mathrm{PGE}_{2}-$ mediated tumor lymphangiogenesis is complex (13), involving many cell types and EP receptors that likely participate in much cross-talk (Figure 1).

In summary, emerging evidence suggests that lymphangiogenesis is a normal part of the inflammatory cycle and constitutes one of many components that serve to return tissues to a normal state of homeostasis after inflammation or remodeling. Immune-suppressive features of such an environment are necessary to avoid local autoimmune reactions and resolve the inflammation. The recent discovery that LECs can scavenge and cross-present exogenous antigen for $\mathrm{T}$ cell deletion $(2,14)$ is consistent with a role for these cells in restoration of tissue homeostasis and prevention of autoimmunity. Finally, because tumors induce a microenvironment with hallmarks of immune-suppressive chronic inflammation, it is likely that tumor lymphangiogenesis promotes tumor escape of host immunity.

\section{Lactation, involution, and breast cancer}

Among the many risk factors in metastatic breast cancer are pregnancy and lactation history due to the important changes induced both hormonally as well as physically in the breast tissue. In multiple studies and meta-analyses, lactation has been correlated with reduced breast cancer risk, especially in genetically predisposed women (15). On the other hand, cancers that develop in women within 5 years of childbirth have an increased risk of metastasis and mortality compared with those that develop later. When lactation ends, dramatic changes in the breast occur to remodel the milk-producing ducts back into a quiescent state in a process termed involution. This remodeling of the breast tissue has been a major focus of the Schedin lab, who demonstrated previously that various features of the inflammatory environment accompanying involution drive cancer susceptibility and in particular promote more invasive and prometastatic cancer development in rodents (16, 17). Specifically, these features include collagen remodeling, which contributes to a stiffer extracellular matrix, an established risk factor for disease (18); alternatively activated macrophages, which promote immune suppression; and COX-2 expression, which drives the synthesis of $\mathrm{PGE}_{2}$ that in turn affects the immune microenvironment in the many ways described above. Based on these results, the Schedin group has suggested that inhibiting inflammation during the involution period, for example, with NSAIDs, may constitute a window of opportunity to prevent metastatic breast cancer by preventing the resulting collagen synthesis and immunesuppressive features that are known to drive cancer progression.

In this issue, Lyons et al. take this notion one step further and demonstrate that lymphangiogenesis accompanies the tissue remodeling that occurs during postpartum mammary duct involution, a process that requires $\mathrm{PGE}_{2}$ (10). Because lymphangiogenesis is part of the inflammatory remodeling program, the results of Lyons and colleagues suggest that pharmacological inhibition of $\mathrm{PGE}_{2}$ signaling during the involution period could potentially help prevent postpartum metastatic breast cancer by inhibiting lymphangiogenesis. In support of this notion, COX-2 inhibition during involution both blocked lymphangiogenesis and prevented metastasis in murine models. These exciting findings provide further rationale for antiinflammatory treatment during lactation weaning.

Many questions remain and will need to be addressed before therapeutic strategies can be designed and clinically implemented. First, how is the normal physiological process of involution affected by inhibiting the inflammatory processes that drive this remodeling? Can involution proceed normally if COX-2 is inhibited, and would the resulting breast return to a normal quiescent state? Second, as lymphangiogenesis and other COX-2driven events contribute immune-suppressive functions in the tissue, would autoimmune reactions be a risk of attenuating this program?

Finally, it will be important to determine whether lymphangiogenesis actually drives the more invasive breast cancer in these patients or merely correlates with the inflammation that supports its onset. Would specifically blocking lymphangiogenesis during this weaning period give the same efficacy as more generally inhibiting all COX-2-dependent inflammatory processes? Lymphangiogenesis can be inhibited specifically by antibody-mediated blockade of VEGFR-3, which prevents LEC proliferation but does not destroy preexisting lymphatic vessels (19). Such inhibitors have been widely shown in mouse models to prevent metastasis, although their effects on the overall tumor immune microenvironment are poorly understood. Such function-blocking antibodies might also be interesting potential targets in the prophylactic context of the targeted window during involution.

\section{Acknowledgments}

The Swartz lab is funded in part by grants from the European Research Council (AdG-323053), the Swiss National Science Foundation (31003A_153471), the Swiss Cancer League (KFS 3312-08-2013), and Swiss TransMed.

Address correspondence to: Melody A. Swartz, 929 E. 57th St., Gordon Center for Integrative Science, W432, Chicago, Illinois 60637, USA. Phone: 773.702.0452; E-mail: melodyswartz@uchicago.edu. 
1. Aspelund A, Alitalo K. Lymphangiogenic factors, mechanisms, and applications. JClin Invest. 2014;124(3):878-887.

2. Lund AW, et al. VEGF-C promotes immune tolerance in B16 melanomas and cross-presentation of tumor antigen by lymph node lymphatics. Cell Rep. 2012;1(3):191-199.

3. Swartz MA, Lund AW. Lymphatic and interstitial flow in the tumour microenvironment: Linking mechanobiology with immunity. Nat Rev Cancer. 2012;12(3):210-219.

4. Huggenberger R, et al. An important role of lymphatic vessel activation in limiting acute inflammation. Blood. 2011;117(17):4667-4678.

5. Kalinski P. Regulation of immune responses by prostaglandin E2. J Immunol. 2012;188(1):21-28.

6. Issa A, Le TX, Shoushtari AN, Shields JD, Swartz MA. Vascular endothelial growth factor-C and C-C chemokine receptor 7 in tumor celllymphatic cross-talk promote invasive phenotype. Cancer Res. 2009;69(1):349-357.

7. Su JL, et al. Cyclooxygenase-2 induces EP1- and HER-2/Neu-dependent vascular endothelial growth factor-C up-regulation: A novelmechanism of lymphangiogenesis in lung adenocarcinoma. Cancer Res. 2004;64(2):554-564.
8. Timoshenko AV, Chakraborty C, Wagner GF, Lala PK. COX-2-mediated stimulation of the lymphangiogenic factor VEGF-C in human breast cancer. Br J Cancer. 2006; 94(8):1154-1163.

9. Xin XP, Majumder M, Girish GV, Mohindra V, Maruyama T, Lala PK. Targeting COX-2 and EP4 to control tumor growth, angiogenesis, lymphangiogenesis and metastasis to the lungs and lymph nodes in a breast cancer model. Lab Invest. 2012;92(8):1115-1128.

10. Lyons TR, et al. Cyclooxygenase-2-dependent lymphangiogenesis promotes nodal metastasis of postpartum breast cancer. JClin Invest. 2014;124(9):3901-3912.

11. Miyata Y, et al. Tumor-associated stromal cells expressing E-prostanoid 2 or 3 receptors in prostate cancer: correlation with tumor aggressiveness and outcome by angiogenesis and lymphangiogenesis. Urology. 2013;81(1):136-142.

12. Kubo H, et al. Host prostaglandin EP3 receptor signaling relevant to tumor-associated lymphangiogenesis. Biomed Pharmacother. 2010;64(2):101-106.

13. Karnezis T, Shayan R, Fox S, Achen MG, Stacker $\mathrm{SA}$. The connection between lymphangiogenic signalling and prostglandin biology: A missing link in the metastatsic pathway. Oncotarget. 2012;3(8):893-906.

14. Hirosue S, et al. Steady-state antigen scavenging, cross-presentation, and CD8(+) T cell priming: A new role for lymphatic endothelial cells. Jimmunol. 2014;192(11):5002-5011.

15. Faupel-Badger JM, et al. Postpartum remodeling, lactation, and breast cancer risk: summary of a National Cancer Institute-sponsored workshop. J Natl Cancer Inst. 2013;105(3):166-174.

16. Lyons TR, et al. Postpartum mammary gland involution drives progression of ductal carcinoma in situ through collagen and COX-2. Nat Med. 2011;17(9):1109-1115.

17. O'Brien J, et al. Alternatively activated macrophages and collagen remodeling characterize the postpartum involuting mammary gland across species. Am J Pathol. 2010;176(3):1241-1255.

18. Yu HM, Mouw JK, Weaver VM. Forcing form and function: biomechanical regulation of tumor evolution. Trends Cell Biol. 2011;21(1):47-56.

19. Pytowski $P$, et al. Complete and specific inhibition of adult lymphatic regeneration by a novel VEGFR-3 neutralizing antibody.

J Natl Cancer Inst. 2005;97(1):14-21. 